\title{
DNA 碱基与高氯酸根共吸附行为的表面增强拉曼光谱研究
}

\author{
崔 丽 1 , 任 斌 2 , * 田中群 2 \\ ('中国科学院城市环境研究所, 福建 厦门 $361021 ; 2$ 厦门大学化学化工学院化学系, \\ 固体表面物理化学国家重点实验室, 福建 厦门 361005)
}

\begin{abstract}
摘要：共吸附有助于实现弱吸附分子或离子的高灵敏表面增强拉曼光谱(SERS)检测. 本文研究了四种脱氧核 糖核酸(DNA)碱基, 即腺嘌呤、鸟嘌呤、胞嘧啶、胸腺嘧啶与高氯酸根 $\left(\mathrm{ClO}_{4}^{-}\right)$在金纳米粒子表面的共吸附行为, 并 考察了吸附能力、电位、共存阴离子等因素的影响. 研究发现四种碱基在质子化后都可以与 $\mathrm{ClO}_{4}^{-}$发生共吸附, 但 在金表面吸附能力弱的胸腺嘧啶与 $\mathrm{ClO}_{4}^{-}$共吸附所获得的 $\mathrm{ClO}_{4}^{-}$信号最弱. 另外, 负电位下电极的排斥作用, 以及 较正电位下基底 SERS 增强效应减小等因素都会导致 $\mathrm{ClO}_{4}^{-}$信号衰减. 此外, $\mathrm{Cl}^{-} 、 \mathrm{NO}_{3}^{-} 、 \mathrm{SO}_{4}^{2-}$ 等阴离子可以与 $\mathrm{ClO}_{4}^{-}$ 发生可逆动态竞争共吸附, 同时引起 $\mathrm{ClO}_{4}^{-}$信号减弱. 以上结果将为提高共吸附法检测弱吸附离子的灵敏度提供 重要参考.
\end{abstract}

关键词：共吸附；表面增强拉曼光谱；DNA 碱基；高氯酸根 中图分类号: $\mathrm{O} 647$

\section{Surface-Enhanced Raman Spectroscopic Study on the Co-adsorption of DNA Bases with Perchlorate}

\author{
CUI Li ${ }^{1, *} \quad$ REN Bin ${ }^{2, *} \quad$ TIAN Zhong-Qun ${ }^{2}$ \\ ('Institute of Urban Environment, Chinese Academy of Sciences, Xiamen 361021, Fujian Province, P. R. China; \\ ${ }^{2}$ State Key Laboratory of Physical Chemistry of Solid Surfaces, Department of Chemistry, College of Chemistry and \\ Chemical Engineering, Xiamen University, Xiamen 361005, Fujian Province, P. R. China)
}

\begin{abstract}
Co-adsorption with molecules that strongly adsorb on metallic surfaces is beneficial in improving the surfaceenhanced Raman spectroscopy (SERS) detection sensitivity of some weakly adsorbed molecules or ions. In this paper, the co-adsorption of four deoxyribonucleic acid (DNA) bases: adenine, guanine, cytosine, and thymine with $\mathrm{ClO}_{4}^{-}$was studied. Factors like adsorption ability, applied potentials, and coexisting anions were examined to illustrate how they affect co-adsorption. We found that the four DNA bases in their protonated forms could co-adsorb with $\mathrm{ClO}_{4}^{-}$. Among them, thymine with the weakest adsorption ability on $\mathrm{Au}$ showed the weakest co-adsorption ability with $\mathrm{ClO}_{4}^{-}$. In addition, repulsion from both negative potentials and reduced SERS enhancements of Au substrates at relatively positive potentials led to a decrease in the SERS intensity of $\mathrm{ClO}_{4}^{-}$. Moreover, anions such as $\mathrm{Cl}^{-}, \mathrm{NO}_{3}^{-}$, and $\mathrm{SO}_{4}^{2-}$ were shown to influence the co-adsorption of $\mathrm{ClO}_{4}^{-}$with adenine based on a dynamic and reversible competitive co-adsorption process. The above results are very helpful in optimizing the SERS detection sensitivity of weakly adsorbed ions or molecules via the co-adsorption method.
\end{abstract}

Key Words : Co-adsorption; Surface-enhanced Raman spectroscopy； DNA bases; $\mathrm{ClO}_{4}^{-}$

表面增强拉曼光谱( surface-enhanced Raman spectroscopy, SERS) 是一种具有极高检测灵敏度的
表面检测技术, 目前已达到痕量甚至单分子检测水 平 ${ }^{[1]}$, 同时, 水的干扰小, 可使苂光淬灭, 适于表面研

Received: August 12, 2009; Revised: September 23, 2009; Published on Web: December 7, 2009.

*Corresponding authors. Email: bren@xmu.edu.cn, lcui@iue.ac.cn; Tel: +86-592-2186532, +86-592-6190534.

The project was supported by the National Natural Science Foundation of China $(20673086,20620130427,20825313)$ and Natural Science Foundation of Fujian Province, China (2009J05032).

国家自然科学基金(20673086, 20620130427,20825313)和福建省自然科学基金(2009J05032)资助项目

C. Editorial office of Acta Physico-Chimica Sinica 
究等特点使得 SERS 的应用领域不断拓宽, 目前已 遍及化学、环境科学、生物医学等诸多领域 ${ }^{[2-7]}$. 但是, SERS 技术的增强效应和高检测灵敏度需要分子能 够吸附在金属纳米结构表面, 因此, 巨大增强效应通 常只发生在吸附能力强的分子上. 而对于一些吸附 能力弱的分子或离子如 $\mathrm{ClO}_{4}^{-}$, SERS 信号通常很弱 甚至检测不到, 因而无法实现 SERS 的直接检测. 然 而, 借助共吸附的方法, 即在纳米粒子表面首先修饰 吸附能力强的分子, 利用其与弱吸附分子的相互作 用, 如静电或氢键作用, 将弱吸附分子拉近纳米结 构表面, 可使其拉曼信号获得有效增强 ${ }^{[5-6,8-12]}$. 高氯 酸盐在环境中广泛存在并具有严重危害性. 它是一 种强力甲状腺毒素, 干扰人体的甲状腺功能, 导致发 育迟缓, 对胎儿和婴儿大脑发育造成影响, 目前, 作 为固体火箭燃料成分之一的高氯酸盐已经对美国 22 个州的供水造成了污染. 另外, 美国七个品牌的 婴儿配方奶粉中也发现了高氯酸盐 ${ }^{[13-14]}$. 在我国, 有 大量以高氯酸钾作为直接产品的化工厂, 而在生产 中利用高氯酸铵作为氧化添加剂的厂家更是数不 胜数 ${ }^{[15]}$. 共吸附方法已被成功用于弱吸附离子高氯 酸根 $\left(\mathrm{ClO}_{4}^{-}\right)$的 SERS 高灵敏度检测 ${ }^{[5,8]}$. 目前, 已有多 种分子被证实可通过静电或氢键作用与 $\mathrm{ClO}_{4}^{-}$发 生共吸附, 如硫脲 (thiourea)及其衍生物甲基硫脲 (methyl thiourea)和烯丙基硫脲(allyl thiourea)、胱氨 二盐酸盐(cystamine dihydrochloride)、2-二甲氨基乙 硫醇盐酸盐(2-dimethylaminoethanethiol hydrochloride)、咪唑(imidazole)等 ${ }^{[5,8,16-18]}$. Gu 等和在利用共吸附 提高 $\mathrm{ClO}_{4}^{-}$检测灵敏度方面开展了一系列工作, 他们 发现利用胱氨二盐酸盐与 $\mathrm{ClO}_{4}^{-}$的共吸附, $\mathrm{ClO}_{4}^{-}$的 SERS 检测限可达到 $5 \times 10^{-6} \mathrm{~mol} \cdot \mathrm{L}^{-1[5]}$, 如果换用 2 二甲氨基乙硫醇盐酸盐与 $\mathrm{ClO}_{4}^{-}$的共吸附, 检测限 可达到 $1 \times 10^{-9} \mathrm{~mol} \cdot \mathrm{L}^{-1}$, 并且 SERS 信号强度与浓度 存在良好的线性关系 ${ }^{[8]}$, 实现了实际受污染水体中的 $\mathrm{ClO}_{4}^{-}$浓度的定量分析, 并获得了与常规的离子色谱 方法接近的浓度值. 但相对于离子色谱, 表面增强 拉曼光谱(SERS)更有望实现快速、灵敏、无损伤的 在线检测 ${ }^{[5,8]}$. 另外共吸附的方法也已用于其它污染 离子的检测, 如放射性离子 $\mathrm{TcO}_{4}^{-}$和 $\mathrm{UO}_{2}^{2+}{ }^{2}$ 等 $^{[6,9]}$, 检测 限分别达到 $1 \times 10^{-7} \mathrm{~mol} \cdot \mathrm{L}^{-1}$ 和 $8 \times 10^{-7} \mathrm{~mol} \cdot \mathrm{L}^{-1}$. 虽然共 吸附方法在定量分析方面获得了很好的结果, 但影 响检测效果的因素, 如为何两种分子对共吸附的 $\mathrm{ClO}_{4}^{-}$的检测灵敏度存在三个数量级以上的巨大差 别, 其它阴离子如何影响以及为何 $\mathrm{ClO}_{4}^{-}$会被优先共
吸附等, 仍未获得清楚认识.

随着 SERS 技术应用领域的拓宽, 更多的物质 有可能需要借助共吸附的方法实现检测. 为了使共 吸附方法获得更广阔的应用, 我们需要对共吸附行 为进行深人认识, 了解其中的影响因素, 并发展出更 多环境友好型分子实施共吸附行为. 虽然硫脲与 $\mathrm{ClO}_{4}^{-}$的共吸附获得了广泛认识 ${ }^{[10-11,16,19]}$, 但由于硫嫝 对植物、动物、水体和土壤微生物等具有毒性作用, 对臭氧层也具有一定的危险性 ${ }^{[20]}$, 因此不是一种环 境友好型分子, 不利于以后的实际应用. 为此, 本文 研究了四种 DNA 碱基与 $\mathrm{ClO}_{4}^{-}$的共吸附行为, 相比 之下, 碱基分子对环境的危害较小. 并且考察了电 位、吸附能力、其它共存阴离子对共吸附的影响, 为 共吸附方法的发展和共吸附分子的选择提供参考.

\section{1 实 验 \\ 1.1 试 剂}

高氯酸钠 $\left(\mathrm{NaClO}_{4}, 98.0 \%-102.0 \%\right.$, Alfa Aesar)、 腺嘌呤 $\left(\mathrm{C}_{5} \mathrm{H}_{5} \mathrm{~N}_{5}, 99 \%\right.$, Alfa Aesar $)$ 、鸟嘌呤 $\left(\mathrm{C}_{5} \mathrm{H}_{5} \mathrm{~N}_{5} \mathrm{O},>\right.$ $99 \%$, Acros $) 、$ 胞嘧啶 $\left(\mathrm{C}_{4} \mathrm{H}_{5} \mathrm{~N}_{3} \mathrm{O},>98 \%\right.$, Alfa Aesar $) 、$ 胸腺嘧啶 $\left(\mathrm{C}_{5} \mathrm{H}_{6} \mathrm{~N}_{2} \mathrm{O}_{2}, 99 \%, \mathrm{Sigma}\right)$, 氯金酸 $\left(\mathrm{HAuCl}_{4} \cdot\right.$ $\left.4 \mathrm{H}_{2} \mathrm{O}\right)$ 及柠檬酸纳 $\left(\mathrm{C}_{6} \mathrm{H}_{5} \mathrm{Na}_{3} \mathrm{O}_{7} \cdot 2 \mathrm{H}_{2} \mathrm{O}\right)$ 为分析纯, 国药 集团化学试剂有限公司提供.

\subsection{SERS 基底制备}

金纳米粒子的合成采用 Frens 的方法 ${ }^{[2]}$, 即将 $100 \mathrm{~mL} 0.01 \%$ 的氯金酸溶液加热至沸后, 搅拌的同 时加人 $0.65 \mathrm{~mL}$ 浓度为 $1 \%$ 的柠檬酸钠溶液还原, 制 备出的金粒子粒径约 $60 \mathrm{~nm}$. 将金纳米粒子离心清 洗三次后, 将离心浓缩的金粒子滴在抛光的金电极 上, 空气中干燥后备用.

\section{3 仪 器}

拉曼光谱实验使用 LabRam I 型共焦显微拉曼 光谱仪(法国 Dilor 公司), 激发线波长为 $632.8 \mathrm{~nm}$, 物 镜是 Olympus $50 \times$ 的长焦镜头(数值孔径 0.55 , 工作 距离 $8 \mathrm{~mm}$ ). 电化学原位拉曼检测使用 XHD-II 型 恒电位仪(厦门大学)控制电位. 文中所给出的电位 均相对于饱和甘采电极.

\section{2 结果与讨论}

\section{1 吸附能力对 $\mathrm{ClO}_{4}^{-}$共吸附的影响}

首先研究腺嘌呤与 $\mathrm{ClO}_{4}^{-}$的共吸附, 图 1 曲线 $\mathrm{a}$ 是 中性溶液 $1 \mathrm{mmol} \cdot \mathrm{L}^{-1}$ 腺嘌呤 $+0.1 \mathrm{~mol} \cdot \mathrm{L}^{-1} \mathrm{NaClO}_{4}$ 中 腺嘌呤吸附在金上的 SERS 谱, 从中观察不到 $\mathrm{ClO}_{4}^{-}$ 


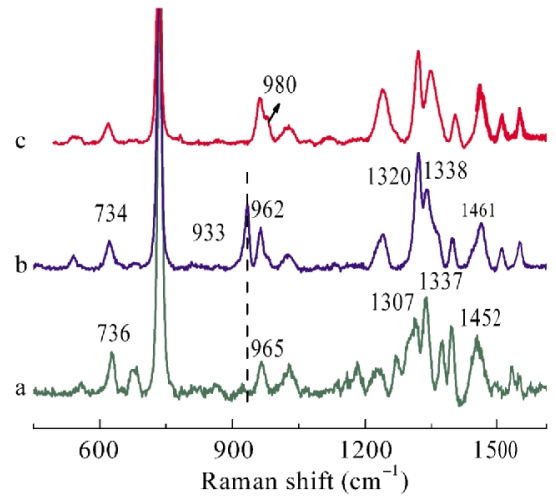

图 1 不同溶液和 $\mathrm{pH}$ 值条件下腺嘌呤和 $\mathrm{ClO}_{4}^{-}$的 SERS 谱

Fig.1 SERS spectra of adenine and $\mathrm{ClO}_{4}^{-}$in solutions with different $\mathrm{pH}$ values and anions

(a) $1 \mathrm{mmol} \cdot \mathrm{L}^{-1}$ adenine $+0.1 \mathrm{~mol} \cdot \mathrm{L}^{-1} \mathrm{NaClO}_{4}$; (b) $1 \mathrm{mmol} \cdot \mathrm{L}^{-1}$ adenine $+0.1 \mathrm{~mol} \cdot \mathrm{L}^{-1} \mathrm{HClO}_{4}$; (c) $1 \mathrm{mmol} \cdot \mathrm{L}^{-1}$ adenine+ $0.1 \mathrm{~mol} \cdot \mathrm{L}^{-1} \mathrm{HClO}_{4}+0.5 \mathrm{~mol} \cdot \mathrm{L}^{-1} \mathrm{H}_{2} \mathrm{SO}_{4}$

位于 $933 \mathrm{~cm}^{-1}$ 的特征峰. 将溶液换成 $\mathrm{pH}=1$ 的酸性 溶液 $\left(1 \mathrm{mmol} \cdot \mathrm{L}^{-1}\right.$ 腺嘌呤 $\left.+0.1 \mathrm{~mol} \cdot \mathrm{L}^{-1} \mathrm{HClO}_{4}\right)$, 由于 此 $\mathrm{pH}$ 值低于质子化腺嘌呤的 $\mathrm{p} K_{\mathrm{a}}$ 值 $(3.8)^{[22]}$, 腺嘌呤 发生质子化, 此时我们观察到在 $933 \mathrm{~cm}^{-1}$ 处出现了 一个新峰(图 1 曲线 b), 这个峰与 $\mathrm{ClO}_{4}^{-}$的全对称振动 峰的位置吻合. 由于质子化后, 腺嘌呤谱峰的位置和 相对强度与中性溶液相比发生了较大变化, 为了验 证 $933 \mathrm{~cm}^{-1}$ 处来自 $\mathrm{ClO}_{4}^{-}$, 而不是腺嘌呤质子化后由 于构型改变产生的新峰, 我们在溶液中加人数滴 0.5 $\mathrm{mol} \cdot \mathrm{L}^{-1} \mathrm{H}_{2} \mathrm{SO}_{4}$, 图 1 曲线c是此情况下获得的 SERS 谱图. 可以清楚看出, $933 \mathrm{~cm}^{-1}$ 峰消失, 同时在 980 $\mathrm{cm}^{-1}$ 出现 $\mathrm{SO}_{4}^{2-}$ 的特征峰. 由于 $\mathrm{SO}_{4}^{2-}$ 与 $\mathrm{ClO}_{4}^{-}$同为阴离 子, 都会与质子化后带正电的腺嘌呤发生共吸附, 这 个现象说明 $\mathrm{SO}_{4}^{2-}$ 取代了 $\mathrm{ClO}_{4}^{-}$导致 $933 \mathrm{~cm}^{-1}$ 峰消失, 同时也证明了 $933 \mathrm{~cm}^{-1}$ 处峰来自 $\mathrm{ClO}_{4}^{-}$, 而不是质子
化腺嘌呤.

对于其它三种 DNA 碱基: 鸟嘌呤、胞嘧啶和胸 腺嘧啶, 我们也同样比较了中性和酸性溶液中的情 况, 如图 2 所示. 可以看出, 在这三种 DNA 碱基 $\mathrm{pH}=1$ 酸性溶液中, 在 $933 \mathrm{~cm}^{-1}$ 出现清楚的 $\mathrm{ClO}_{4}^{-}$振 动峰, 说明在 $\mathrm{pH}=1$ 条件下碱基发生质子化, 以静电 作用与 $\mathrm{ClO}_{4}^{-}$发生共吸附. 对比这四种 DNA 碱基, 发 现酸性条件下与胸腺嘧啶发生共吸附的 $\mathrm{ClO}_{4}^{-}$的信 号最不明显. 文献中已有对 DNA 碱基在金上吸附 能力进行详细研究的报道 ${ }^{[23-26]}$, 发现胸腺嘧啶在四 种碱基中的吸附能力最弱, 导致其不能有效地与 $\mathrm{ClO}_{4}^{-}$发生共吸附. 以上情况说明吸附能力强的分子 对 $\mathrm{ClO}_{4}^{-}$的共吸附效果更好, 这也说明 $\mathrm{Gu}$ 等 ${ }^{[5,8]}$ 选用 含颈基的分子作为共吸附分子研究 $\mathrm{ClO}_{4}^{-}$的 SERS 检测灵敏度是合理的.

另外, 在腺嘌呤的中性溶液中, 我们观测不到任 何关于 $\mathrm{ClO}_{4}^{-}$的信号, 而在其它三种碱基的中性溶液 中, 尤其是胞嘧啶和胸腺嘧啶中, 我们仍然可以看到 弱的 $\mathrm{ClO}_{4}^{-}$的信号. 与不含碱基分子的 $\mathrm{NaClO}_{4}$ 溶液 中的表面谱比较, 发现这些信号实际来自溶液中 $\mathrm{ClO}_{4}^{-}$的贡献, 由于浓度高 $\left(0.1 \mathrm{~mol} \cdot \mathrm{L}^{-1}\right)$, 当碱基的 SERS 信号强度不是足够强的时候, 仍然可以观察 到 $\mathrm{ClO}_{4}^{-}$的信号. 腺嘌呤的 SERS 信号非常强, 所以 溶液中 $\mathrm{ClO}_{4}^{-}$的信号在谱图中观察不到, 而胞嘧啶和 胸腺嘧啶的 SERS 信号不是很强, 所以溶液中 $\mathrm{ClO}_{4}^{-}$ 的弱信号仍然能观测到.

\section{2 电位对 $\mathrm{ClO}_{4}^{-}$共吸附影响}

为了更全面理解共吸附行为, 我们研究了电极 电位对共吸附行为的影响. 图 3 是中性和酸性溶液 中腺嘌呤吸附在金电极上随电位变化的 SERS 谱.
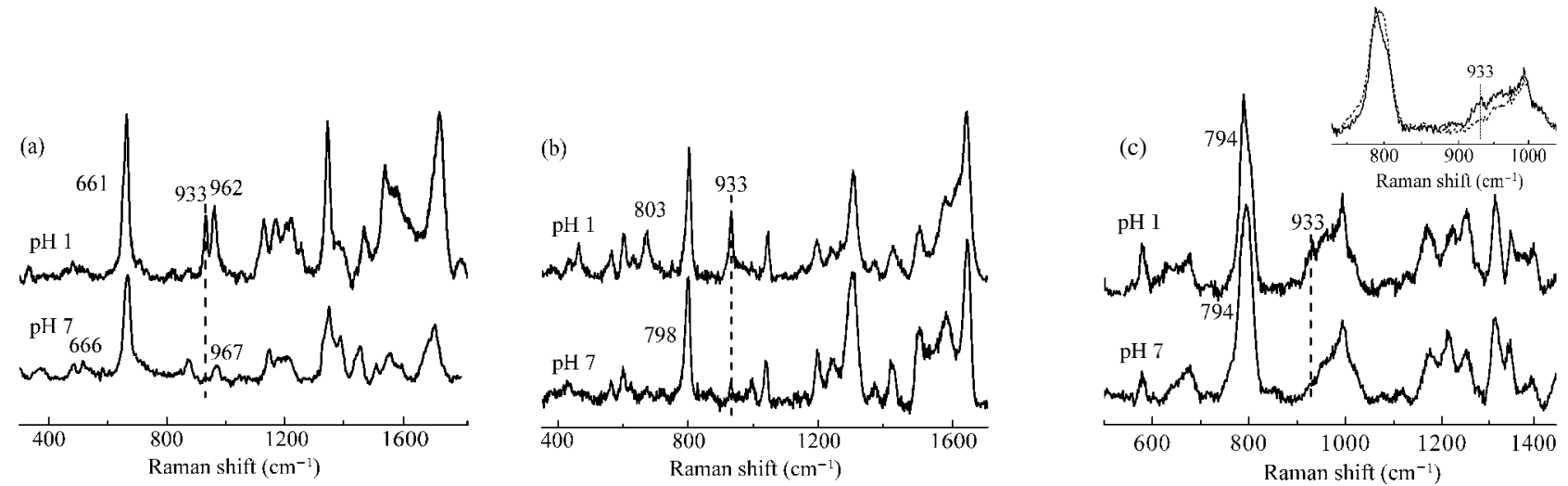

图 2 乌嘌呤 (a)、胞嘧啶(b)、胸腺嘧啶(c)在酸性和中性溶液中在金表面上的 SERS 谱

Fig.2 SERS spectra of guanine (a), cytosine (b), and thymine (c) on gold surfaces in acid and neutral solutions (a) $1 \mathrm{mmol} \cdot \mathrm{L}^{-1}$ guanine $+0.1 \mathrm{~mol} \cdot \mathrm{L}^{-1} \mathrm{ClO}_{4}^{-}$; (b) $1 \mathrm{mmol} \cdot \mathrm{L}^{-1}$ cytosine $+0.1 \mathrm{~mol} \cdot \mathrm{L}^{-1} \mathrm{ClO}_{4}^{-}$; (c) $10 \mathrm{mmol} \cdot \mathrm{L}^{-1}$ thymine $+0.1 \mathrm{~mol} \cdot \mathrm{L}^{-1} \mathrm{ClO}_{4}^{-}$ 

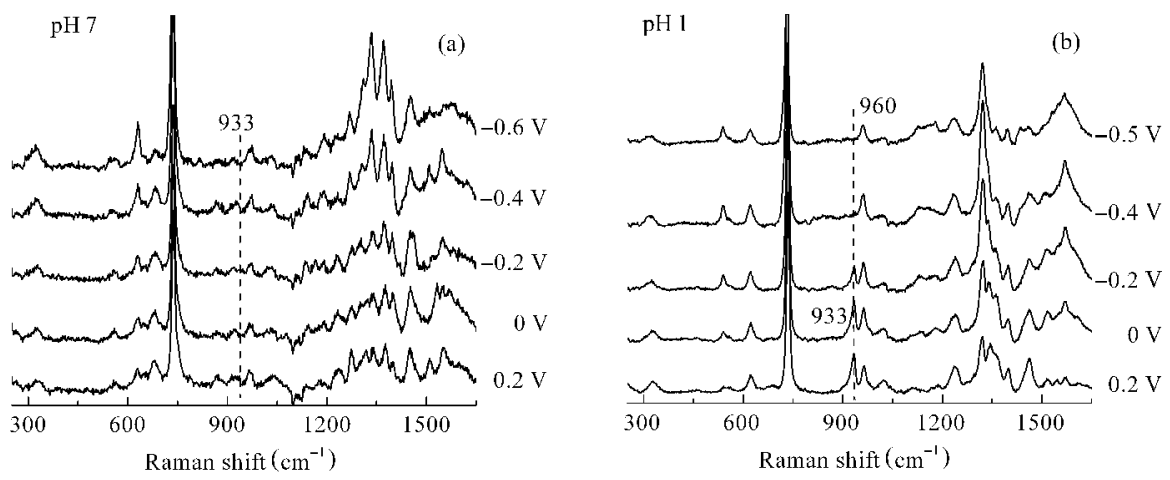

图 3 中性和酸性溶液中腺嘌呤在金表面在不同电位下的 SERS 谱

Fig.3 Potential dependent SERS spectra of adenine adsorbed on gold surfaces in neutral and acid solutions

(a) $1 \mathrm{mmol} \cdot \mathrm{L}^{-1}$ adenine $+0.1 \mathrm{~mol} \cdot \mathrm{L}^{-1} \mathrm{NaClO}_{4}$; (b) $1 \mathrm{mmol} \cdot \mathrm{L}^{-1}$ adenine $+0.1 \mathrm{~mol} \cdot \mathrm{L}^{-1} \mathrm{HClO}_{4}$

在中性腺嘌呤溶液中, 当电位从开路电位 $0.2 \mathrm{~V}$ 负 移至 $-0.6 \mathrm{~V}$ 时, 虽然腺嘌呤的信号随电位负移而增 强, 但 $\mathrm{ClO}_{4}^{-}$的信号始终观察不到. 在酸性腺嘌呤溶 液中, 随电位从正往负, 腺嘌呤的信号增强, 在电位 到达析氢区后, 由于腺嘌呤脱附而信号减弱. 而 $\mathrm{ClO}_{4}^{-}$, 其信号随电位负移逐渐减弱, 并在 $-0.4 \mathrm{~V}$ 时彻底消 失. 这种情况说明负电位下电极表面对 $\mathrm{ClO}_{4}^{-}$有排斥 作用, 当这种作用大于质子化腺嘌呤与 $\mathrm{ClO}_{4}^{-}$的静电 力时, $\mathrm{ClO}_{4}^{-}$将离开电极表面而导致信号观察不到. 但是, 这并不意味电位越正越好, 因为电位越正, 基 底的增强效应通常会减小, SERS 信号因此变弱. 如 图4(b)酸性溶液中鸟嘌呤随电位变化的SERS谱图, $0.2 \mathrm{~V}$ 下, 不仅鸟嘌呤信号弱于 $0 \mathrm{~V}$ 时的, $\mathrm{ClO}_{4}^{-}$的信 号也弱于 $0 \mathrm{~V}$ 时的. 中性溶液中, 鸟嘌呤的情况与腺 嘌呤相同, 即在 $0.4 \mathrm{~V}$ 到 $-0.6 \mathrm{~V}$ 电位区间, $\mathrm{ClO}_{4}^{-}$的信 号始终未出现. 以上情况说明, 电位宜选在较正的电 位, 但要根据 SERS 信号确定最佳值.

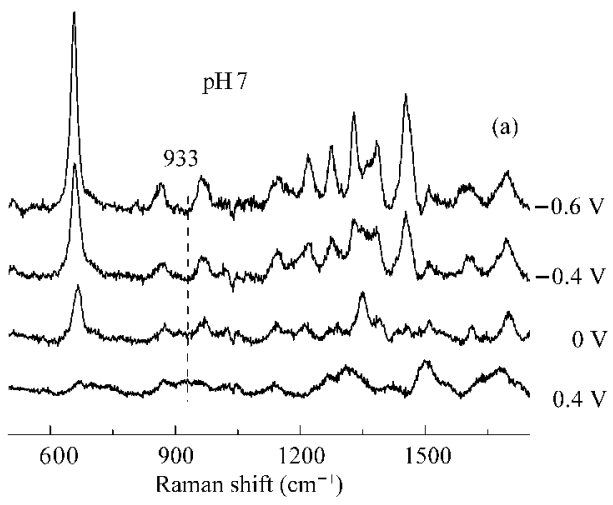

\section{3 其它共存阴离子对 $\mathrm{ClO}_{4}^{-}$共吸附的影响}

为了研究其它共存阴离子对 $\mathrm{ClO}_{4}^{-}$信号的影响, 我们固定体系的 $\mathrm{pH}$ 值、 $\mathrm{ClO}_{4}^{-}$和腺嘌呤浓度, 研究与 $\mathrm{ClO}_{4}^{-}$相同浓度的 $\mathrm{Cl}^{-} 、 \mathrm{NO}_{3}^{-} 、 \mathrm{SO}_{4}^{2-}$ 的存在对 $\mathrm{ClO}_{4}^{-}$信号 强度的影响. 如图 5(A)所示, 在不更换电极的情况 下, 当将溶液从 $0.1 \mathrm{~mol} \cdot \mathrm{L}^{-1} \mathrm{HClO}_{4}+1.5 \mathrm{mmol} \cdot \mathrm{L}^{-1}$ 腺 嘌呤换成 $0.1 \mathrm{~mol} \cdot \mathrm{L}^{-1} \mathrm{HCl}+0.1 \mathrm{~mol} \cdot \mathrm{L}^{-1} \mathrm{NaClO}_{4}+1.5$ $\mathrm{mmol} \cdot \mathrm{L}^{-1}$ 腺嘌呤后, 位于 $933 \mathrm{~cm}^{-1}$ 的 $\mathrm{ClO}_{4}^{-}$峰的信号 减弱, 说明部分 $\mathrm{Cl}^{-}$置换了 $\mathrm{ClO}_{4}^{-}$, 但是当将溶液再换 回 $0.1 \mathrm{~mol} \cdot \mathrm{L}^{-1} \mathrm{HClO}_{4}+1.5 \mathrm{mmol} \cdot \mathrm{L}^{-1}$ 腺嘌呤后, $\mathrm{ClO}_{4}^{-}$ 峰的信号变强, 并基本恢复到原有水平, 可逆性良 好. 在 $\mathrm{HNO}_{3}$ 溶液中的情况与 $\mathrm{HCl}$ 中类似, 如图 5(B) 所示, 位于 $933 \mathrm{~cm}^{-1} \mathrm{ClO}_{4}^{-}$峰减弱, 同时在 $1042 \mathrm{~cm}^{-1}$ 出现 $\mathrm{NO}_{3}^{-}$峰, 当溶液换回 $0.1 \mathrm{~mol} \cdot \mathrm{L}^{-1} \mathrm{HClO}_{4}+1.5$ $\mathrm{mmol} \cdot \mathrm{L}^{-1}$ 腺嘌呤后, $\mathrm{ClO}_{4}^{-}$峰基本恢复. 但加人 $\mathrm{H}_{2} \mathrm{SO}_{4}$ 溶液后, 如图 5(C)所示, $933 \mathrm{~cm}^{-1}$ 峰基本观察不到, 而 $980 \mathrm{~cm}^{-1}$ 峰变强. 当溶液换回 $0.1 \mathrm{~mol} \cdot \mathrm{L}^{-1} \mathrm{HClO}_{4}+$

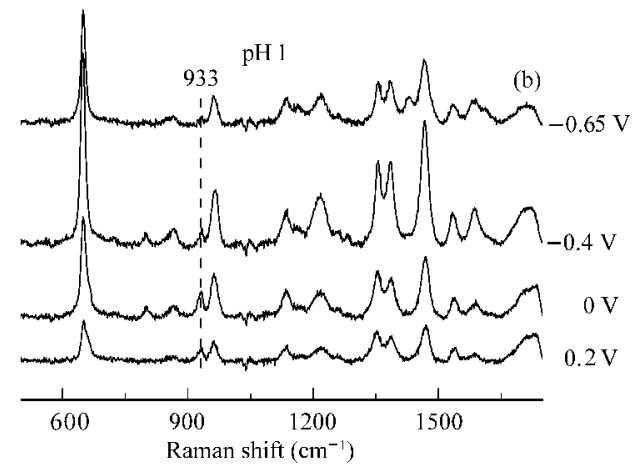

图 4 中性和酸性溶液中鸟嘌呤在不同电位下的 SERS 谱

Fig.4 Potential dependent SERS spectra of guanine in neutral and acid solutions (a) $1 \mathrm{mmol} \cdot \mathrm{L}^{-1}$ guanine $+0.1 \mathrm{~mol} \cdot \mathrm{L}^{-1} \mathrm{NaClO}_{4}$; (b) $1 \mathrm{mmol} \cdot \mathrm{L}^{-1}$ guanine $+0.1 \mathrm{~mol} \cdot \mathrm{L}^{-1} \mathrm{HClO}_{4}$ 

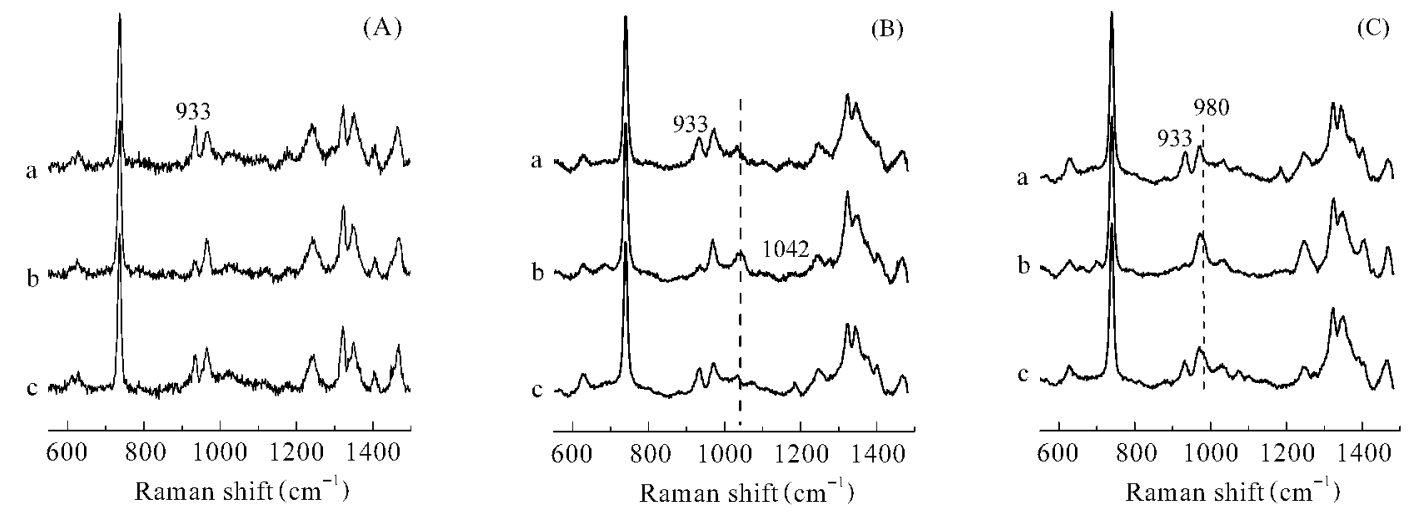

图 5 腺嘌呤在不同溶液中的 SERS 谱

Fig.5 SERS spectra of adenine in different solutions

$1.5 \mathrm{mmol} \cdot \mathrm{L}^{-1}$ adenine $+0.1 \mathrm{~mol} \cdot \mathrm{L}^{-1} \mathrm{ClO}_{4}^{-}\left(\mathrm{A}-\mathrm{C}\right.$, a , c) with different competitive anions: $(\mathrm{A}, \mathrm{b}) 0.1 \mathrm{~mol} \cdot \mathrm{L}^{-1} \mathrm{HCl}$,

(B, b) $0.1 \mathrm{~mol} \cdot \mathrm{L}^{-1} \mathrm{HNO}_{3},(\mathrm{C}, \mathrm{b}) 0.1 \mathrm{~mol} \cdot \mathrm{L}^{-1} \mathrm{H}_{2} \mathrm{SO}_{4}$

$1.5 \mathrm{mmol} \cdot \mathrm{L}^{-1}$ 腺嘌呤, $933 \mathrm{~cm}^{-1}$ 峰不能完全恢复到初 始值. 以上结果说明, 其它阴离子同样与质子化腺嘌 呤存在共吸附, 并且会与 $\mathrm{ClO}_{4}^{-}$发生竞争共吸附, 二 价 $\mathrm{SO}_{4}^{2-}$ 比 $\mathrm{NO}_{3}^{-}$或 $\mathrm{Cl}^{-}$对 $\mathrm{ClO}_{4}^{-}$共吸附的影响大, 但这 种竞争共吸附是动态的, 且具有较好的可逆性.

在 $\mathrm{Gu}$ 等 ${ }^{[16]}$ 研究胱氨二盐酸盐和 2 -二甲氨基乙硫 醇盐酸盐与 $\mathrm{ClO}_{4}^{-}$的共吸附时也发现, 在 $\mathrm{Cl}^{-} 、 \mathrm{NO}_{3}^{-} 、 \mathrm{SO}_{4}^{2-}$ 、

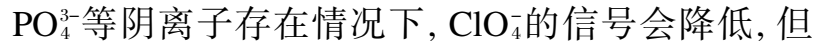
$\mathrm{ClO}_{4}^{-}$仍然是优先共吸附离子, 原因是当其它阴离子 的浓度比 $\mathrm{ClO}_{4}^{-}$浓度高 $2-3$ 个数量级时, $\mathrm{ClO}_{4}^{-}$的信号仍 然比其它阴离子强. 但在研究硫脲与多种阴离子共 吸附行为时发现, $\mathrm{SO}_{4}^{2-}$ 相比于 $\mathrm{ClO}_{4}^{-}$是优先共吸附离 子. 两种不同的结论除了可能与共吸附分子相关外, 还可能与样品制备方法不同有关. 本文与后者都是 在电化学体系中进行(即固/液界面), 都发现 $\mathrm{SO}_{4}^{2-}$ 比 $\mathrm{NO}_{3}^{-}$或 $\mathrm{Cl}^{-}$对 $\mathrm{ClO}_{4}^{-}$共吸附的影响更大. 但在前人的工 作中, 未对竞争的动态过程进行研究, 本文通过更换 阴离子溶液, 发现阴离子的竞争共吸附是动态过程 且具有良好的可逆性.

从以上结果可以看出, 为了提高 $\mathrm{ClO}_{4}^{-}$的检测灵 敏度, 应在保证腺嘌呤分子质子化的前提下, 使用含 一价阴离子的酸, 并尽量减少高价酸的使用, 因为酸 根离子的竞争共吸附会使 $\mathrm{ClO}_{4}^{-}$的信号强度降低. 另 外, 样品的制备方法也应该是需考虑的因素.

\section{3 结 论}

研究了四种 DNA 碱基, 即腺嘌呤、鸟嘌呤、胞 嘧啶、胸腺嘧啶, 在酸性条件下与 $\mathrm{ClO}_{4}^{-}$的共吸附行 为. 研究发现, 四种碱基在质子化后都可以与 $\mathrm{ClO}_{4}^{-}$ 发生共吸附, 并且共吸附与分子的吸附能力、电位、
阴离子等因素有关.

(1) 吸附能力. 在四种碱基中, 胸腺嘧啶与 $\mathrm{ClO}_{4}^{-}$ 共吸附获得的 $\mathrm{ClO}_{4}^{-}$的 SERS 信号最差, 原因主要是 由于胸腺嘧啶在金上的吸附能力较其它三种碱基 弱. 这说明在选择共吸附分子时, 吸附能力强的分子 是更好的选择.

(2) 电位. $\mathrm{ClO}_{4}^{-}$的信号强度随电位负移, 由于电 极的排斥作用, 发生脱附而导致信号衰减. 但电位并 不是越正越好, 在更正的电位, 基底的增强效应减 弱, 同样会导致分子的 SERS 信号减弱. 因此, 在选 择电位时, 应在保证 SERS 信号强度的情况下, 选择 较正电位.

(3) 阴离子. 研究了 $\mathrm{Cl}^{-} 、 \mathrm{NO}_{3}^{-} 、 \mathrm{SO}_{4}^{2-}$ 对 $\mathrm{ClO}_{4}^{-}$的影 响, 发现三种阴离子会与 $\mathrm{ClO}_{4}^{-}$发生竞争共吸附, 并 导致 $\mathrm{ClO}_{4}^{-}$信号减弱, 其中 $\mathrm{SO}_{4}^{2-}$ 的影响更大. 但这种 竞争是动态的, 具有良好的可逆性. 这说明在选择 $\mathrm{pH}$ 值时，应在保证碱基分子质子化的前提下，使用 较低浓度的含一价阴离子的酸.

基于以上研究结果, 我们将在未来利用质子化 DNA 碱基与阴离子的共吸附开展一系列检测工作, 优化实验条件, 提高弱吸附离子的 SERS 检测灵 敏度.

\section{References}

1 Pieczonka, N. P. W.; Aroca, R. F. Chem. Soc. Rev., 2008, 37(5): 946

2 Costa, J. C. S.; Sant' Ana, A. C.; Corio, P.; Temperini, M. L. A. Talanta, 2006, 70(5): 1011

3 Goeller, L. J.; Riley, M. R. Appl. Spectrosc., 2007, 61(7): 679

4 Mahajan, S.; Richardson, J.; Brown, T.; Bartlett, P. N. J. Am. Chem. Soc., 2008, 130(46): 15589 
5 Ruan, C.; Wang, W.; Gu, B. Anal. Chim. Acta, 2006, 567(1): 114

6 Ruan, C.; Luo, W.; Wang, W.; Gu, B. Anal. Chim. Acta, 2007, 605 (1): 80

7 Han, X. X.; Kitahama, Y.; Tanaka, Y.; Guo, J.; Xu, W. Q.; Zhao, B.; Ozaki, Y. Anal. Chem., 2008, 80(17): 6567

8 Gu, B. H.; Ruan, C. M.; Wang, W. Appl. Spectrosc., 2009, 63(1): 98

9 Gu, B.; Ruan, C. Anal. Chem., 2007, 79(6): 2341

10 Cao, P. G.; Yao, J. L.; Ren, B.; Gu, R. N.; Tian, Z. Q. J. Phys. Chem. B, 2002, 106(39): 10150

11 Tian, Z. Q.; Li, W. H.; Mao, B. W.; Gao, J. S. J. Electroanal. Chem., 1994, 379(1-2): 271

12 Tan, S.; Erol, M.; Sukhishvili, S.; Du, H. Langmuir, 2008, 24(9): 4765

13 Gu, B. H.; Coates, J. D. Perchlorate environmental occurence, interactions and treatment. New York: Springer, 2006

14 Kirk, A. B.; Martinelango, P. K.; Tian, K.; Dutta, A.; Smith, E. E.; Dasgupta, P. K. Environ. Sci. Technol., 2005, 39(7): 2011

15 Zhang, K. J.; Gao, Y. N.; Sui, H. M.; Lu, N. Sichuan Environment, 2008, 27(1): 91 [张可佳, 高乃云, 隋铭皓, 卢 宁. 四川环境, 2008, 27(1): 91]

16 Zhong, Q. L.; Wang, D. Q.; Liu, F. M.; Su, X. Q.; Shi, C. H.; Tian, Z. Q. Acta Phys. -Chim. Sin., 1998, 14(6): 562 [钟起玲, 王敦清,
刘峰铭, 粟晓琼, 施财辉, 田中群. 物理化学学报, 1998, 14(6) $562]$

17 Cao, P. G.; Gu, R. A.; Tian, Z. Q. J. Phys. Chem. B, 2003, 107(3): 769

18 Gu, R. A.; Yao, J. L.; Yuan, Y. X.; Zhong, Q. L.; Tian, Z. Q. Acta Chim. Sin., 1998, 56(7): 692 [顾仁敖, 姚建林, 袁亚仙, 钟起玲, 田中群. 化学学报, 1998, 56(7): 692]

19 Zheng, J. Z.; Ren, B.; Wu, D. Y.; Tian, Z. Q. J. Electroanal. Chem., 2005, 574(2): 285

20 http://www.sigmaaldrich.com

21 Frens, G. Nat. Phys. Sci., 1973, 241: 20

22 Puppels, G. J.; Otto, C.; Greve, J.; Robert-Nicoud, M.; Arndt-Jovin, D. J.; Jovin, T. M. Biochemistry, 1994, 33(11): 3386

23 Ostblom, M.; Liedberg, B.; Demers, L. M.; Mirkin, C. A. J. Phys. Chem. B, 2005, 109(31): 15150

24 Kimura-Suda, H.; Petrovykh, D. Y.; Tarlov, M. J.; Whitman, L. J. J. Am. Chem. Soc., 2003, 125(30): 9014

25 Demers, L. M.; Oestblom, M.; Zhang, H.; Jang, N. H.; Liedberg, B.; Mirkin, C. A. J. Am. Chem. Soc., 2002, 124(38): 11248

26 Gourishankar, A.; Shukla, S.; Ganesh, K. N.; Sastry, M. J. Am. Chem. Soc., 2004, 126(41): 13186 